\title{
TRAIL Based Therapy: Overview of Mesenchymal Stem Cell Based Delivery and miRNA Controlled Expression of TRAIL
}

\author{
Rukset Attar ${ }^{1}$, Farhana Sajjad ${ }^{2}$, Muhammad Zahid Qureshi ${ }^{3}$, Fizza Tahir $^{4}$, Ejaz $^{2}$ \\ Hussain $^{5}$, Sundas Fayyaz ${ }^{4}$, Ammad Ahmad Farooqi ${ }^{4 *}$
}

\begin{abstract}
Rapidly increasing number of outstanding developments in the field of TRAIL mediated signaling have revolutionized our current information about inducing and maximizing TRAIL mediated apoptosis in resistant cancer cells. Data obtained with high-throughput technologies have provided finer resolution of tumor biology and now it is known that a complex structure containing malignant cells strictly coupled with a large variety of surrounding cells constitutes the tumor stroma. Utility of mesenchymal stem cells (MSCs) as cellular vehicles has added new layers of information. There is sufficient experimental evidence substantiating efficient gene deliveries into MSCs by retroviral, lentiviral and adenoviral vectors. Moreover, there is a paradigm shift in molecular oncology and recent high impact research has shown controlled expression of TRAIL in cancer cells on insertion of complementary sequences for frequently downregulated miRNAs. In this review we have attempted to provide an overview of utility of TRAIL engineered MSCs for effective killing of tumor and potential of using miRNA response elements as rheostat like switch to control expression of TRAIL in cancer cells.
\end{abstract}

Keywords: TRAIL, Apoptosis - targeted therapy - signaling cascades

Asian Pac J Cancer Prev, 15 (16), 6495-6497

\section{Introduction}

Research over the years has shown that specific genetic, epigenetic factors, suppression of tumor suppressors, overexpression of oncogenes, loss of apoptosis and increased cell survival underlie breast carcinogenesis. TRAIL has emerged as one amongst the most deeply studied molecules in oncology because of its ability to selectively induce apoptosis in cancer cells and leaving normal cells intact. Increasingly sophisticated information is deepening our understanding about TRAIL mediated signaling and it is now evident that TRAIL signals through death receptors which belong to tumor necrosis factor receptor superfamily that possesses a cytoplasmic death domain (DD). Death inducing signaling complex consisting of FADD and Pro-caspase-8 is formed at the death receptor. cFLIP negatively regulates TRAIL induced signaling by interfering with the activation of caspase-8. Caspase- 8 activates its downstream effector caspase- 3 thus functionalizing extrinsic pathway. Intrinsic pathway is activated via Caspase- 8 mediated processing of Bid into truncated Bid. tBid moves into mitochondrion to promote release of cytochrome c, SMAC/DIABLO, Omi/Htra. Cytochrome c, co-operates with Apaf-1 to form apoptosome, which results in activation of caspase-9.

\section{Mesenchymal Stem Cells for Delivery of TRAIL}

Research over the years has progressively highlighted use of cells as therapeutic carriers and high-throughput technologies have revolutionized delivery systems. Using interdisciplinary approaches, delivery systems are being developed to maximize the efficiency and simultaneously increasing the concentration of the drug in the target region. Increasingly it is being realized that mesenchymal stem cells (MSCs) migrate along with other bone marrow-derived cells to the sites of primary tumor and pre-metastatic niche. It is becoming sequentially more understandable that homing of MSCs to tumours is under extensive research to selectively target tumours. There is a rapidly increasing experimental evidence highlighting engineering of MSCs to deliver TRAIL to induce apoptosis.

There is a direct piece of evidence suggesting that different cancer cell lines respond to specific variants of soluble TRAIL. It has been shown that PancTu1 cells respond better to MSC.sTRAIL(DR4) and colorectal cancer cells, HCT116 respond considerably to MSC. sTRAIL(DR5). Moreover, 5-fluorouracil significantly 
enhanced TRAIL induced growth inhibition in mice xenografted with HCT116 cancer cells (Yu et al., 2013).

Human MSCs transduced with a lentiviral vector encoding TRAIL are reported to be effective against malignant mesothelioma cell lines. MSCs injected into mice either intrapleurally or intravenously were traced within the tumour stroma as evidenced by Immunofluorescence. It has been revealed that intravenously-delivered MSCs incorporate into tumours in greater numbers than intrapleurally-delivered MSCs. TRAIL transduced MSCs induced regression of tumour growth when delivered intravenously (Sage et al., 2014).

Using intracranially implanted medulloblastoma (MB) it has been shown that MSC-S-TRAIL has notably enhanced anti-tumor activity upon pre-treatment with MS-275. In-vitro testing with MS-275 demonstrated that there was an upregulated DR4/DR5 in MS-275 treated TRAIL resistant cancer cell line (Nesterenko et al., 2012).

There was a notable cancer growth inhibition in nude mice subcutaneously injected with rat bone marrow mesenchymal stem cells transduced with a lentiviral vector encoding stTRAIL (Deng et al., 2014). Moreover, Human umbilical cord-derived MSCs (HUMSCs) transduced with lentiviral vector encoding stTRAIL driven by alphafetoprotein (AFP) have also been tested in cancer model. It was shown that intravenously injected HUMSCs migrated to tumor site and expressed TRAIL (Yan et al., 2014).

sTRAIL encoding Herpes Simplex Virus (HSV) type I amplicon vector has also been used to engineer TRAIL expressing MSCs. Mechanistically it was revealed that carbenoxolone (CBX) increased TRAIL induced apoptosis via upregulation of DR5 and downregulation of connexin 43. It has been noted that TRAIL and CBX increased the survival of treated mice by $27 \%$ in an intracranial glioma model (Yulyana et al, 2013).

There is an exciting piece of evidence highlighting combinatorial approach using chemotherapeutic agent temozolomide and MSC-based TRAIL gene therapy. Results revealed that temozolomide considerably improved sensitivity to TRAIL-induced apoptosis. It was shown that temozolomide modulated expression of DR5 via ERK. Additionally temozolomide exerted its inhibitory effects on anti-apoptotic proteins including XIAP and cFLIP (Kim et al., 2014).

Dodecameric TRAIL(dTRAIL) and herpes simplex virus thymidine kinase (HSV-TK) carrying MSCs have also shown considerable potential. It has been experimentally verified that HSV-TK converts ganciclovir (GCV) into a toxic triphosphorylated form, that consequently induced apoptosis. MSC/dTRAIL-TK significantly induced regression of metastatic tumors and prolonged survival of xenografted mice. MSC migratory potential was evaluated and results revealed that a greater number of MSCs was found in the vicinity of tumor nodule sites compared with non-nodule sites. It was reported that sequential administrations of MSC/dTRAIL-TK $(5 \times 105$ cells) in 2 -week intervals (with double and triple injections) considerably enhanced survival of xenografted mice (Kim et al., 2013).

In-vitro analysis has shown that CD20-specific TRAIL fusion protein induced apoptosis in CD20-positive BJAB cells, however, it was not effective in CD20-negative Jurkat cells. MSCs have also been used to deliver CD20specific TRAIL fusion protein in NOD/SCID mouse subcutaneous BJAB lymphoma xenograft model (Yan et al, 2013).

MK886, a lipoxygenase inhibitor has been shown to improve TRAIL induced apoptosis in TRAIL resistant human glioma U-251MG and U-373MG cells. Tumor growth in the mice treated with MK886 and MSC-TRAIL was noted to be considerably reduced (Kim et al., 2012).

\section{Diametrically Opposed Roles of Umbilical Cord Blood-Derived MSCs and Adipose Tissue-Derived MSCs}

Rapidly increasing information regarding MSCs mediated delivery of TRAIL to tumor site is enhancing our understanding of efficacy of different MSCs. It has lately been indicated that co-culturing umbilical cord bloodderived MSCs with primary glioblastoma multiforme (GBM) cells inhibited growth of cancer cells. On the contrary, co-culturing adipose-tissue-derived MSCs with GBM induced dramatic increase in cell proliferation. Experimental evidence indicated that developed tumors were highly vascularized when AT-MSCs and GBM were cotransplanted (Akimoto et al., 2013).

\section{miRNA Response Element Controlled Expression of TRAIL}

miRNA are small non-coding RNAs that have emerged as key regulators of wide ranging biological mechanisms. miRNAs have been extensively studied regulators in molecular oncology and it is now evident that tumor suppressor miRNAs are repressed/downregulated and oncogenic miRNAs are overexpressed in cancer cells (Farooqi et al., 2014; Mollaie et al., 2013; Orang et al., 2014). Differential expression of miRNAs has been studied in different cancers and high-throughput technologies have added new layers of information into the existing pool of knowledge (Ding et al., 2014; Diao et al., 2014). Various natural and synthetic agents have been noted to repress and upregulate expression of oncogenic and tumor suppressor miRNAs respectively (Zhou et al., 2014).

TRAIL related gene therapy and how five of recently published articles have opened new horizons for differential expression of TRAIL using previously existing knowledge about dysregulated miRNAs in cancer cells. To selectively induce expression of TRAIL in cancer cells, there are some exciting pieces of evidence indicating that insertion of the complementary sequences for miRNAs selectively suppressed in cancer cells into the expression cassette could enhance the expression of gene of interest in tumor cells.

MREs of miR-143, miR-145 and miR-122 were inserted into adenoviral vectors to control TRAIL expression (Ad-TRAIL-M3). Selective expression of TRAIL in prostate cancer cells by Ad-TRAIL-M3 resulted in considerably enhanced apoptosis. Ad-TRAIL-M3 treated xenografted mice also revealed significantly reduced growth of tumors (Huo et al., 2014). Adenoviral 
vector carrying MREs of miR-143 and miR-122 has also been shown to induce apoptosis via selective expression of TRAIL in esophageal cancer cells (Zhou et al., 2014).

\section{Conclusion}

However, despite considerable advancements in molecular biology, genetically engineered MSCs still contain the intrinsic mechanisms that can induce tumorigenesis, metastasis and drug resistance. Therefore there is a need to re-interpret and re-evaluate the data obtained through in-vitro and in-vivo studies reporting the use of MSCs as vehicles to target tumours.

\section{References}

Akimoto K, Kimura K, Nagano M, et al (2013). Umbilical cord blood-derived mesenchymal stem cells inhibit, but adipose tissue-derived mesenchymal stem cells promote, glioblastoma multiforme proliferation. Stem Cells Dev, 22, 1370-86.

Deng Q, Zhang Z, Feng X, et al (2014). TRAIL-secreting mesenchymal stem cells promote apoptosis in heat-shocktreated liver cancer cells and inhibit tumor growth in nude mice. Gene Ther, 21, 317-27.

Huo W, Jin N, Fan L, Wang W (2014). MiRNA regulation of TRAIL expression exerts selective cytotoxicity to prostate carcinoma cells. Mol Cell Biochem, 388, 123-33.

Kim SM, Woo JS, Jeong CH, et al (2014). Potential application of temozolomide in mesenchymal stem cell-based TRAIL gene therapy against malignant glioma. Stem Cells Transl Med, 3, 172-82.

Kim SW, Kim SJ, Park SH, et al (2013). Complete regression of metastatic renal cell carcinoma by multiple injections of engineered mesenchymal stem cells expressing dodecameric TRAIL and HSV-TK. Clin Cancer Res, 19, 415-27.

Nesterenko I, Wanningen S, Bagci-Onder T, et al (2012). Evaluating the effect of therapeutic stem cells on TRAIL resistant and sensitive medulloblastomas. PLoS One, 7, e49219

Sage EK, Kolluri KK, McNulty K, et al (2014). Systemic but not topical TRAIL-expressing mesenchymal stem cells reduce tumour growth in malignant mesothelioma. Thorax, 69, 638-47.

Yan C, Li S, Li Z, et al (2013). Human umbilical cord mesenchymal stem cells as vehicles of CD20-specific TRAIL fusion protein delivery: a double-target therapy against nonHodgkin's lymphoma. Mol Pharm, 10, 142-51.

Yan C, Yang M, Li Z, et al (2014). Suppression of orthotopically implanted hepatocarcinoma in mice by umbilical cordderived mesenchymal stem cells with sTRAIL gene expression driven by AFP promoter. Biomaterials, 35, 3035-43.

Yu R, Deedigan L, Albarenque SM, et al (2013). Delivery of sTRAIL variants by MSCs in combination with cytotoxic drug treatment leads to p53-independent enhanced antitumor effects. Cell Death Dis, 4, e503.

Yulyana Y, Endaya BB, Ng WH, et al (2013).Carbenoxolone enhances TRAIL-induced apoptosis through the upregulation of death receptor 5 and inhibition of gap junction intercellular communication in human glioma. Stem Cells Dev, 22, 1870-82.

Zhou K, Yan Y, Zhao S (2014). Esophageal cancer-selective expression of TRAIL mediated by MREs of miR-143 and miR-122. Tumour Biol, 35, 5787-95.

Kim SM, Woo JS, Jeong CH, et al (2012). Effective combination therapy for malignant glioma with TRAIL-secreting mesenchymal stem cells and lipoxygenase inhibitor MK886. Cancer Res, 72, 4807-17.

Ding H, Wu YL, Wang YX, Zhu FF (2014). Characterization of the microRNA expression profile of cervical squamous cell carcinoma metastases. Asian Pac J Cancer Prev, 15, 1675-9.

Farooqi AA, Qureshi MZ, Coskunpinar E, et al (2014). miR-421, miR-155 and miR-650: emerging trends of regulation of cancer and apoptosis. Asian Pac J Cancer Prev, 15, 1909-12.

Zhou RP, Chen G, Shen ZL, Pan LQ (2014). Cinobufacin suppresses cell proliferation via miR-494 in BGC-823 gastric cancer cells. Asian Pac J Cancer Prev, 15, 1241-5.

Diao CY, Guo HB, Ouyang YR, et al (2014). Screening for metastatic osteosarcoma biomarkers with a DNA microarray. Asian Pac J Cancer Prev, 15, 1817-22.

Orang AV, Safaralizadeh R, Hosseinpour Feizi MA (2014). Insights into the Diverse Roles of miR-205 in Human Cancers. Asian Pac J Cancer Prev, 15, 577-83.

Mollaie HR, Monavari SH, Arabzadeh SA, et al (2013). RNAi and miRNA in viral infections and cancers. Asian Pac J Cancer Prev, 14, 7045-56. 